\title{
Early urinary biomarkers for renal tubular damage in spontaneously hypertensive rats on a high salt intake
}

\author{
Keiko Hosohata ${ }^{1}$, Daisuke Yoshioka ${ }^{1}$, Akira Tanaka ${ }^{2}$, Hitoshi Ando ${ }^{1}$ and Akio Fujimura ${ }^{1}$
}

A high salt intake exacerbates hypertension and accelerates renal tubular damage in hypertensive patients. However, data concerning early biomarkers for renal tubular change induced by a high salt intake are limited. The objective of this study was to clarify the time course of new biomarkers for renal tubular damage during high salt intake in spontaneously hypertensive rats (SHR). Male SHR received a regular or high-salt diet from 9 to 17 weeks of age. At 10 weeks of age, a high salt intake caused renal tubular damage, which was further exacerbated at 17 weeks of age. Although albuminuria was detected in salt-loaded SHR at 14 weeks of age, urinary excretion of vanin-1 and neutrophil gelatinase-associated lipocalin (NGAL) was elevated in these animals from 10-17 weeks of age. However, kidney injury molecule-1 (Kim-1) was elevated at 15 weeks of age in salt-loaded SHR. These results suggest that urinary vanin-1 and NGAL are potentially early biomarkers for renal tubular damage in SHR under a high salt intake.

Hypertension Research (2016) 39, 19-26; doi:10.1038/hr.2015.103; published online 17 September 2015

Keywords: early biomaker; high salt intake; hypertension; renal tubular damage

\section{INTRODUCTION}

A high salt intake can markedly elevate blood pressure (BP) in hypertensive patients, ${ }^{1}$ which is supported by clinical data showing that a reduction in salt intake causes a significant fall in BP in patients with essential hypertension. ${ }^{2,3}$ Elevated BP increases the risk for cardiovascular and cerebrovascular morbidity and mortality. ${ }^{4}$ In addition to its influence on BP, salt promotes organ damage, leading to cardiovascular ${ }^{5,6}$ and cerebrovascular ${ }^{7}$ diseases.

Renal injury is also caused by a high salt intake. Clinical studies have demonstrated that a high salt intake promotes albuminuria ${ }^{6}$ and a decline in glomerular filtration rate $^{8}$ in hypertensive patients. However, data regarding the profiles of renal tubular damage during the onset of renal injury caused by high salt intake are limited in these patients. $^{9}$

Recently, several new biomarkers for renal tubular damage have been identified. For example, increased urinary excretion of kidney injury molecule-1 (Kim-1) ${ }^{10-12}$ and neutrophil gelatinase-associated lipocalin (NGAL), ${ }^{13,14}$ have been shown to reflect proximal tubular damage. We also found that urinary excretion of vanin-1, which is an epithelial glycosylphosphatidylinositol (GPI)-anchored pantetheinase, ${ }^{15,16}$ is elevated before urinary increases of Kim-1 and NGAL in rats with nephrotoxicant- and drug-induced renal tubular injury. ${ }^{17,18}$ These data led us to speculate that, compared with urinary Kim-1 and NGAL, urinary vanin-1 is an early biomarker for renal tubular damage in hypertensive patients. This study is the first step to demonstrate this hypothesis. Time courses of urinary vanin-1, Kim-1 and NGAL were determined in spontaneously hypertensive rats (SHR), a model of human essential hypertension, under high salt intake.

\section{METHODS}

Experimental protocol

All animal procedures were approved by the Animal Care and Use Committee of Jichi Medical University (Shimotsuke, Japan) and were performed in accordance with the Guidelines for Animal Research. Male SHR (Charles River Japan, Yokohama, Japan) were obtained at 8 weeks of age and maintained under a specific pathogen-free condition and controlled temperature and humidity, with a 12-h light/12-h dark cycle $(0700-1900 \mathrm{~h} / 1900-0700 \mathrm{~h})$. The rats were fed a regular diet (CE-2; CLEA Japan, Tokyo, Japan) and water ad libitum for 1 week before experimental use. Nine-week-old rats either were given a high-salt diet or continued to receive the regular diet. We chose an $8 \%$ $\mathrm{NaCl}$ diet, because many previous studies that examined the profiles of saltinduced renal damage used $8 \% \mathrm{NaCl}$ in food. ${ }^{19,20}$ This enabled us to compare the present results with the previous data.

The following experiments were performed:

Experiment 1: at 9 weeks of age, SHR $(n=12)$ were randomized to regular $(0.8 \% \mathrm{NaCl})$ or high-salt $(8 \% \mathrm{NaCl})$ diet groups. Once a week, a urine sample was collected in a metabolic cage for 8 weeks and stored at $-80^{\circ} \mathrm{C}$ until use. Under the prewarmed conscious state, systolic blood pressure (SBP) was measured by the tail-cuff method (KN-210; Natsume Seisakusho, Tokyo, Japan). At 17 weeks of age, animals were anesthetized with pentobarbital sodium $\left(50 \mathrm{mg} \mathrm{kg}^{-1}\right.$ i.p.) to obtain blood and kidney samples for assessing the renal morphology and mRNA expression of renal tubular endocytic receptors.

${ }^{1}$ Departments of Clinical Pharmacolo.gy, School of Medicine, Jichi Medical University, Tochigi, Japan and ${ }^{2}$ Departments of Pathology, School of Medicine, Jichi Medical University, Tochigi, Japan

Correspondence: Dr A Fujimura, Division of Clinical Pharmacology, Department of Pharmacology, School of Medicine, Jichi Medical University, Tochigi 329-0498, Japan.

E-mail: akiofuji@jichi.ac.jp

Received 20 January 2015; revised 12 July 2015; accepted 23 July 2015; published online 17 September 2015 
Experiment 2: at 9 and 10 weeks of age, which were apparently at an early stage of renal damage after salt loading, rats ( $n=4$ at each time point in each group) were killed to determine an early change in renal tubular morphology and/or mRNA expression of renal tubular endocytic receptors.

\section{Histopathology}

Kidney tissues at 9 (experiment 2), 10 (experiment 2) and 17 (experiment 1) weeks of age were fixed in $4 \%$ paraformaldehyde overnight, dehydrated by passing through an ascending ethanol series, and embedded in paraffin wax. The sections $(4 \mu \mathrm{m})$ were stained with hematoxylin and eosin for the assessment of inflammation, with periodic acid-Schiff for the determination of tubular damage, and with Masson's trichrome (LSI Medience Corporation, Tokyo, Japan) for the assessment of interstitial fibrosis. The sections were examined with a light microscope and analyzed using semiquantitative scoring, ranging from grades 0 to 4 (grade 0 , normal; grade 1, mild; grade 2, moderate; grade 3 , severe; and grade 4 , very severe). The grading was applied for typical hypertension-induced kidney lesions such as tubular degeneration and tubular dilatation. For each group, an average score was calculated.

\section{Immunofluorescence staining}

Immunofluorescence staining of the kidney was performed on frozen sections as previously described. ${ }^{17}$ Briefly, sections were incubated with goat anti-vanin1 antibody (1:50, Santa Cruz Biotechnology, Santa Cruz, CA, USA) and goat anti-Kim-1 antibody (1:1000; R\&D Systems, Minneapolis, MN, USA), and then with donkey Cy3-labeled anti-goat IgG, (1:200; Abcam, Cambridge, UK) and Alexa Fluor 488-labeled phalloidin (Life Technologies, Carlsbad, CA, USA). Images were acquired with an AX80 (Olympus, Tokyo, Japan).

\section{Laboratory measurements}

Urinary, serum and renal concentrations of vanin-1, Kim-1 and NGAL protein were measured using an ELISA kit from Uscn Life Science (Cloud-Clone, Houston, TX, USA), R\&D Systems and BioPorto Diagnostics (Gentofte, Denmark), respectively. In brief, urine and blood samples were centrifuged at $1000 \mathrm{~g}$ for $10 \mathrm{~min}$, and their supernatants were used. Tissue lysate was prepared from renal cortices of rats at 17 weeks of age, and $25 \mu \mathrm{g}$ of total protein measured with the BCA protein assay kit (Thermo scientific, Rockford, IL, USA) was loaded onto an ELISA microplate. Urinary albumin was measured with an autoanalyzer by immunonephelometry (MP Biomedicals Japan, Tokyo, Japan). Serum and urinary concentrations of creatinine were determined by the Jaffe method with a commercial kit (Wako Pure Chemical Industries, Osaka, Japan).

\section{RNA extraction and real-time quantitative PCR}

Total RNA isolated from the kidney tissues using an RNeasy Mini Kit (QIAGEN, Valencia, CA, USA) was reverse-transcribed with a PrimeScript RT reagent Kit (Takara Bio, Otsu, Japan). Gene expression was analyzed by real-time PCR, performed using the Applied Biosystems StepOnePlus RealTime PCR system (Life Technologies). Specific sets of primers and TaqMan probes were obtained from Life Technologies. To control for variation in the amount of cDNA available for PCR in the different samples, expression of target sequences was normalized to that of an endogenous control, glyceraldehyde-3-phosphate dehydrogenase (GAPDH). The GenBank accession number, assay ID, and target exon were NM_030827.1, Rn00578067_m1 and 77-78 (megalin); NM_053332.2, Rn00584200_ml, and 39-40 (cubilin); NM_177421.3, Rn00598583_m1, and 4-5 (24p3R); NM_ 173149.1, Rn00597703_m1 and 5-6 (Havcr1); NM_017008.4, Rn99999916_s1 and 3-3 $(G A P D H)$. Data were analyzed using the comparative threshold cycle method.

\section{Statistical analysis}

Data are expressed as the means \pm s.e.m. Comparisons were made by an unpaired $t$-test. For time course studies, data were compared using two-way ANOVA followed by Bonferroni's post hoc analysis. A value of $P<0.05$ was considered statistically significant. All statistical analyses were conducted with GraphPad Prism, version 4.03 (GraphPad Software, San Diego, CA, USA).

\section{RESULTS}

\section{Effects of high salt intake on SBP}

At 9 weeks of age (before the initiation of salt loading), SBP was not significantly different between the groups (Figure 1). SBP in the group on a high-salt diet was significantly higher than that in the group on a regular diet at 10 and 17 weeks of age (Figure 1).

Effects of high salt intake on urinary albumin, creatinine clearance and renal histopathology

As shown in Figure 2a, urinary albumin excretion in the salt-loaded SHR was significantly higher at 14 weeks of age and further increased thereafter. However, a high salt intake had no significant influence on creatinine clearance at 17 weeks of age in these animals. Renal tubular damage such as tubular degeneration and dilatation was slightly induced at 10 and 17 weeks of age in SHR on a regular diet (Figures $2 \mathrm{~b}$ and $\mathrm{c}$ ). These changes were exaggerated in SHR on a high-salt diet. Interstitial inflammation (Figure 2d) and fibrosis (Figure 2e) were marked in SHR on a high-salt diet at 17 weeks of age, whereas these changes were mild in SHR on a high-salt diet at 10 weeks of age and on a regular diet at 17 weeks of age.

\section{Effects of high salt intake on newly developed renal tubular biomarkers}

Salt loading significantly elevated urinary excretions of vanin-1 and NGAL in SHR at 10 weeks of age, which persisted up to the end of the study (Figure 3). Urinary Kim-1 after salt loading was significantly elevated at 15 and 17 weeks of age.

\section{Origin of urinary vanin-1, NGAL and Kim-1}

To clarify the localization of vanin-1 in the kidney, we carried out immunofluorescence analysis of vanin-1. As shown in Figure 4, vanin-
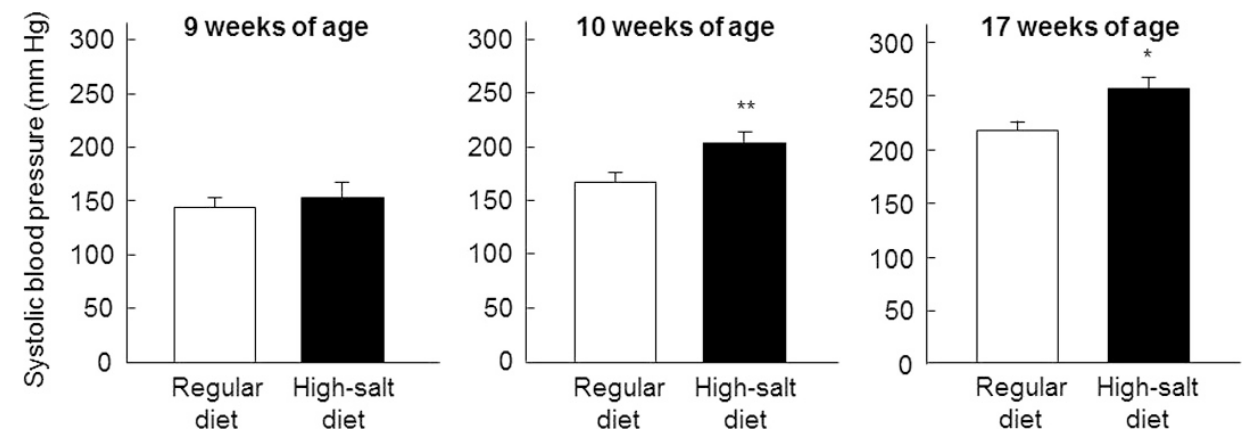

Figure 1 Effects of a high-salt diet on SBP in SHR. SBP was measured by the tail-cuff method in SHR on a regular or high-salt diet from 9 to 17 weeks of age. Data are the means \pm s.e.m. $(n=5-6) .{ }^{*} P<0.05 ;{ }^{* *} P<0.01$ vs. SHR on a regular diet. 
1 expression was detected in the tubules but not the glomeruli, which is consistent with our previous findings. ${ }^{17,18}$ These results indicate that urinary vanin-1 is derived from renal tubules. Of note, the cortex was positively stained for vanin-1 at 9,10 and 17 weeks of age, and the medulla was also stained at 17 weeks of age, which was accompanied by the progression of injury to the medulla, as shown in Figure 4.

We also investigated the origins of urinary NGAL and Kim-1 in this study. At 17 weeks of age, serum and renal concentrations of NGAL protein were detected in each group, and there were no significant differences between the groups (Figure 5a). Because NGAL is reabsorbed in the proximal tubules via endocytic receptors including megalin, cubilin ${ }^{21}$ and $24 \mathrm{p} 3 \mathrm{R},{ }^{22}$ we further investigated the influence of a high-salt diet on these receptors. A high-salt diet significantly reduced the renal mRNA expression of megalin and cubilin at 10 and 17 weeks, respectively, in SHR (Figure 5b). Renal 24p3R mRNA expression was not influenced by high salt intake.
A high-salt diet significantly elevated Kim-1 both in the serum and in the kidney in SHR (Figure 6a). Therefore, it remained probable that urinary Kim-1 was derived from the blood as well as the kidneys. To determine whether urinary Kim-1 was derived from the kidneys, we conducted an immunofluorescence analysis. As shown in Figure 6b, Kim-1 was mainly expressed on the apical side of proximal tubule epithelia in the salt-loaded SHR at 17 weeks of age. These results indicate that urinary Kim-1 originated in the kidney. Consistently with these results, the mRNA expression of renal Kim-1 in the salt-loaded SHR was $\sim 80$-fold higher than that in the controls at 17 weeks of age, whereas there was no significant difference between the groups at 10 weeks of age (Figure 6c).

\section{DISCUSSION}

In this study, the following changes were detected in SHR: (1) a high salt intake caused histologically renal tubular damage, which was
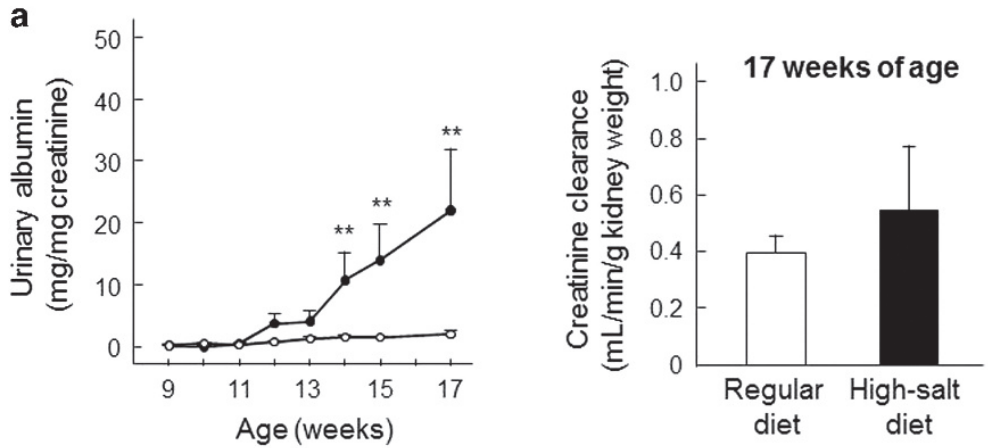

b

9 weeks of age

10 weeks of age
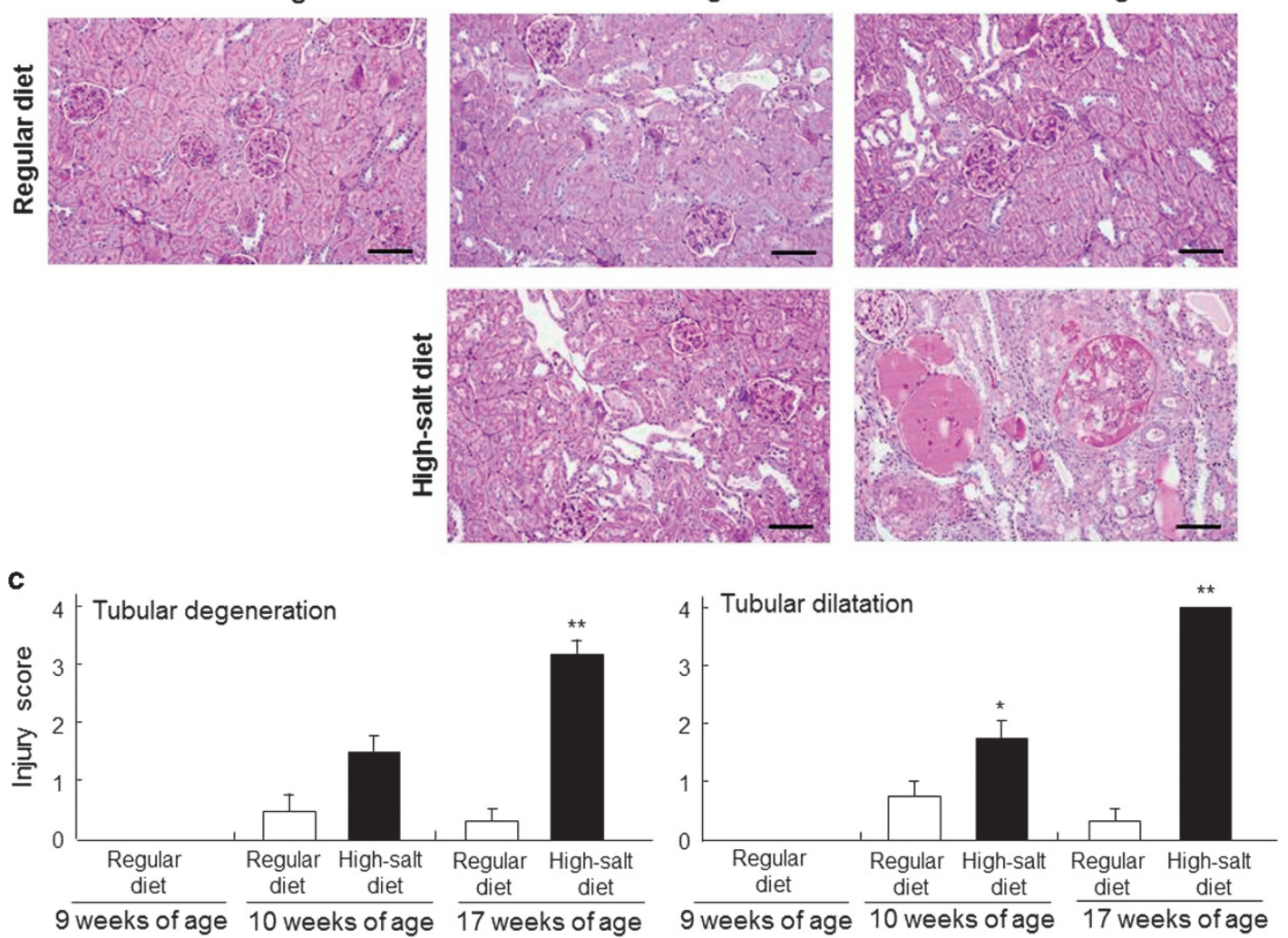

Figure 2 Continued. 
d

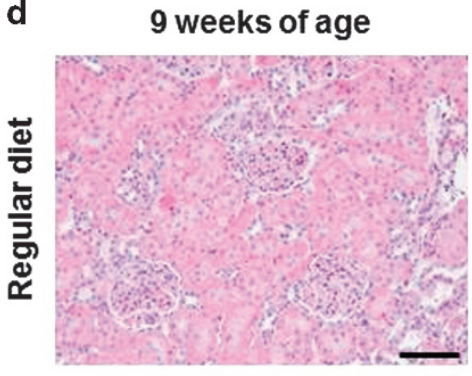

10 weeks of age

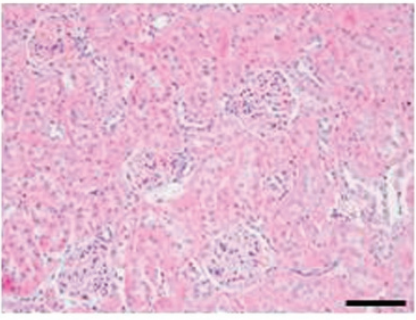

흠

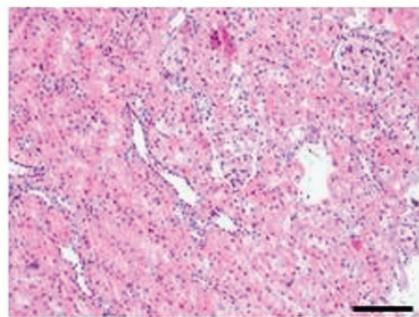

17 weeks of age
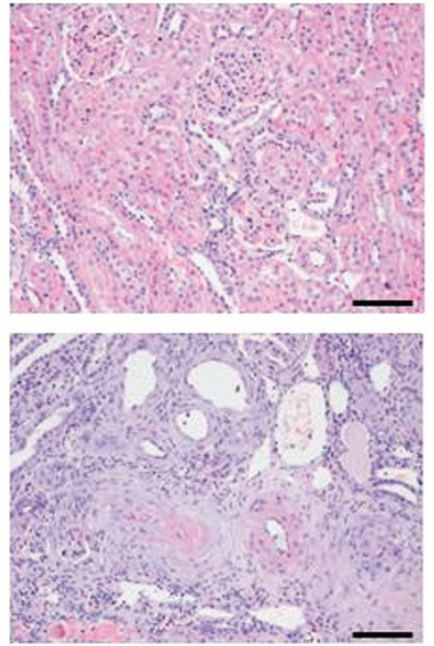

e
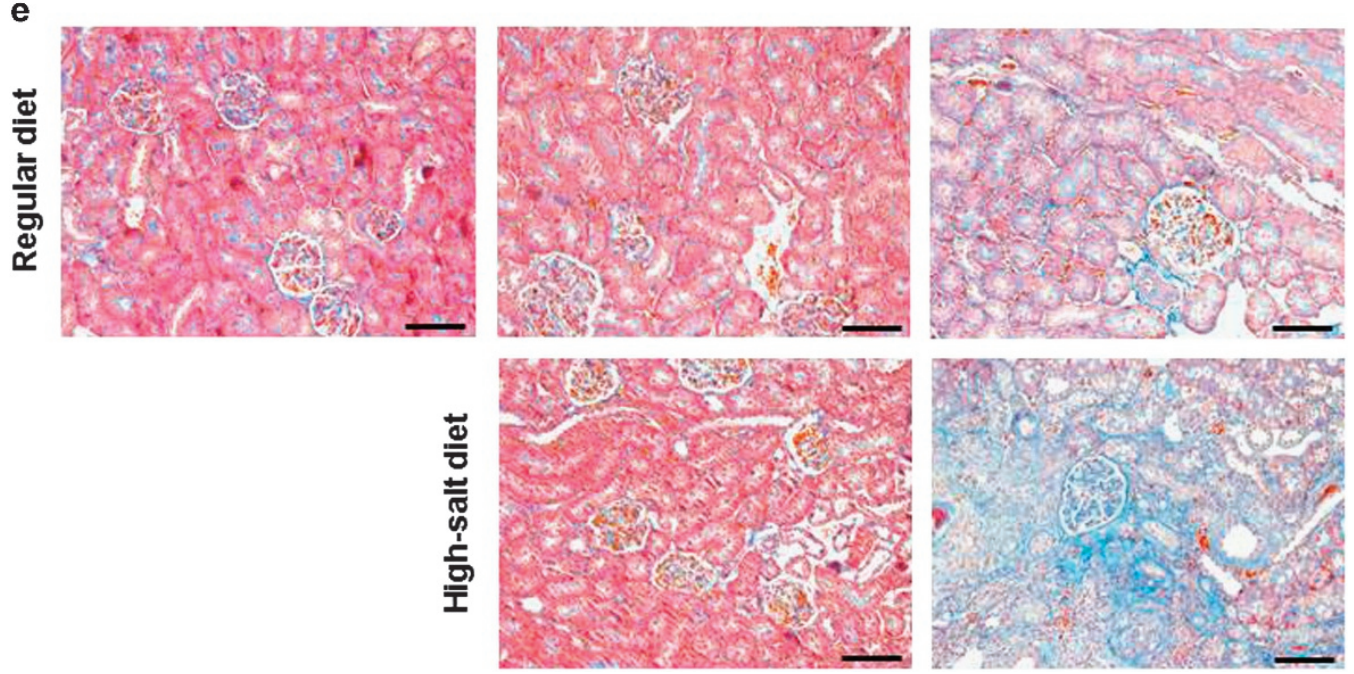

Figure 2 Effects of a high-salt diet on urinary albumin, creatinine clearance, and renal histopathology. (a) Twelve-hour urine samples of SHR on a regular diet (white circles) or a high-salt diet (black circles) were collected once a week from 9 to 17 weeks of age. Urinary albumin concentration was normalized to urinary creatinine concentration. Blood samples were taken from animals at 17 weeks of age. Data are the means \pm s.e.m. $(n=5-6)$. ${ }^{* *} P<0.01$ vs. SHR on a regular diet. (b) Representative photomicrographs of renal changes. Sections were stained by periodic acid-Schiff and hematoxylin. Scale bars, $100 \mu \mathrm{m}$. (c) Renal tubular injury scores. Data are the means \pm s.e.m. $(n=5-6) .{ }^{*} P<0.05 ; * * P<0.01$ vs. SHR on a regular diet. (d, e) Representative photomicrographs of inflammation by hematoxylin and eosin stain (d) and fibrosis by Masson's trichrome stain (e). Scale bars, $50 \mu \mathrm{m}$.
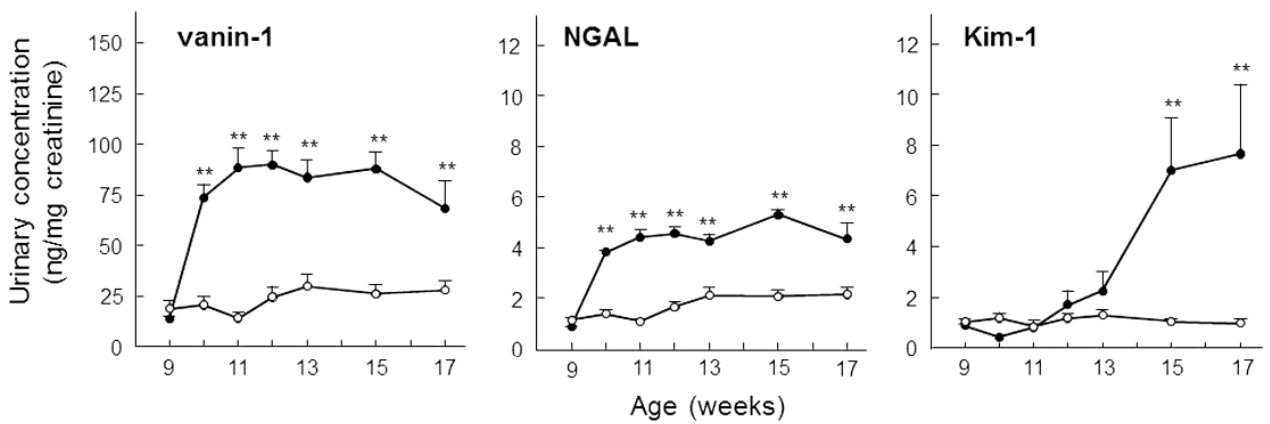

Figure 3 Effects of a high-salt diet on newly developed renal tubular biomarkers. Time course of urinary vanin-1, NGAL and Kim-1. Twelve-hour urine samples of SHR on a regular diet (white circles) or a high-salt diet (black circles) were collected once a week. Concentrations of these markers in urine were determined by ELISA and normalized to urinary creatinine concentration. Data are the means \pm s.e.m. $(n=5-6) . * *<0.01$ vs. SHR on a regular diet. 

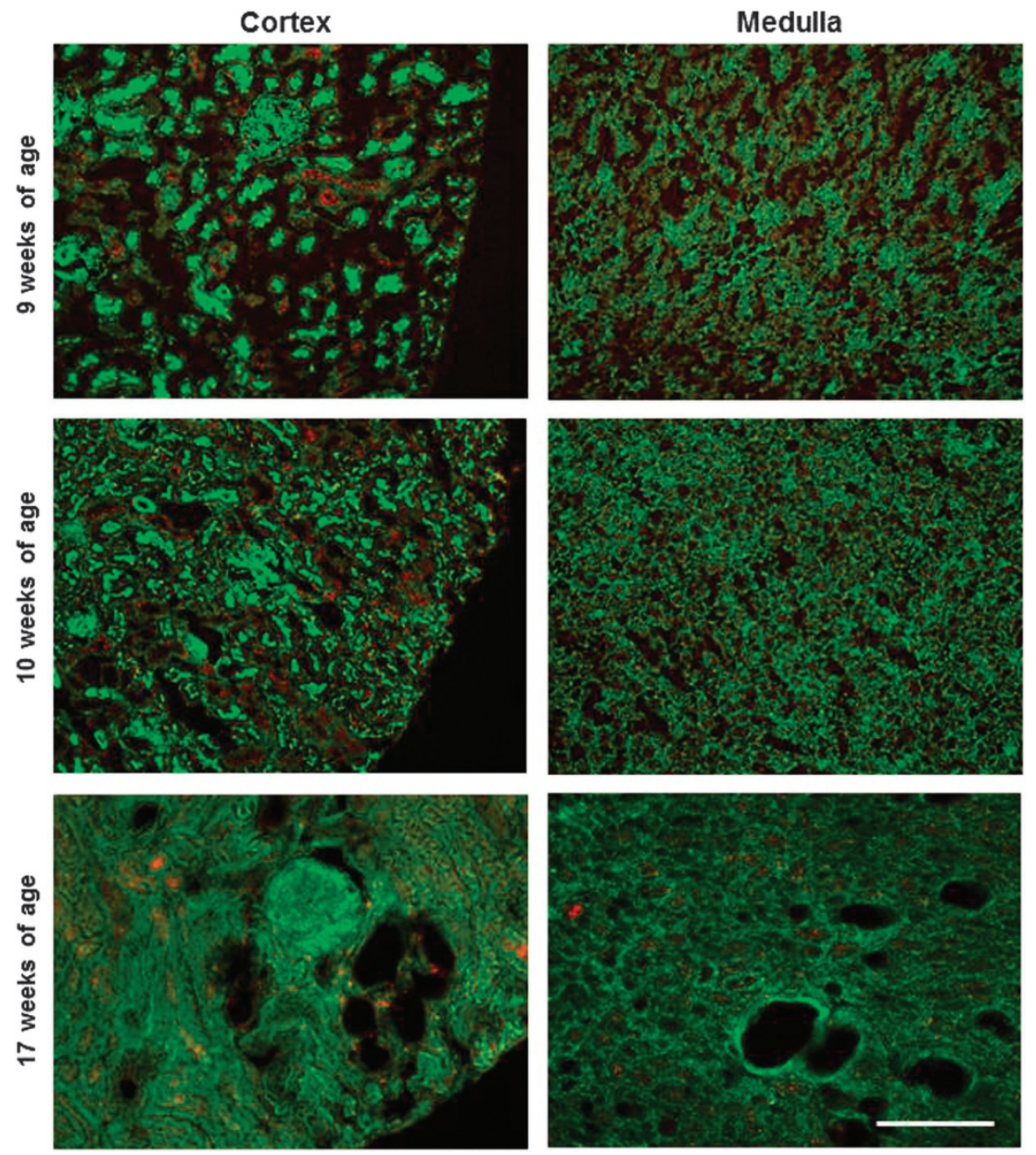

Figure 4 Origin of urinary vanin-1. A representative image of immunostaining of vanin- 1 in the kidney of SHR on a high-salt diet. Cryosections $(4 \mu \mathrm{m})$ were stained with an antibody specific for vanin-1 (red) and phalloidin (green). Scale bars, $200 \mu \mathrm{m}$.

detected before an appearance of albuminuria; (2) urinary excretion of vanin-1 and NGAL was elevated at 1 week after salt loading; and (3) urinary Kim-1 showed a significant increase at 6 weeks after salt loading. These data suggest that, in addition to urinary vanin-1, NGAL is potentially an earlier biomarker for salt-induced renal tubular damage than urinary Kim-1 in SHR, which differs from the previous findings that urinary vanin-1 is elevated before the increases in urinary Kim-1 and NGAL occur in rats with nephrotoxicant- and druginduced renal tubular injury. ${ }^{17,18}$ The differences in the time course of urinary vanin-1, NGAL and Kim-1 might be partly explained by the following mechanisms. After salt loading, oxidative stress causes renal damage. ${ }^{23}$ Lai et al. ${ }^{24}$ have shown that increased reactive oxygen species has a role in salt-induced renal damage in mice. Vanin-1 has been shown to be induced by reactive oxygen species in vivo. ${ }^{25}$ Because reactive oxygen species are involved in the onset of the inflammation process, vanin-1 seems to be induced in the early phase of injury in the salt-loaded SHR. In this study, urinary NGAL appeared early in saltloaded SHR. Of note, the present results clearly demonstrated that a high-salt diet significantly reduces the renal mRNA expression of megalin in the SHR at 10 weeks of age. Because megalin is involved in the endocytic system, it is possible that urinary NGAL could be attributed to the defect in tubular reabsorption. However, urinary Kim-1 increased later in the course of the damage, partly because Kim-1 is upregulated during the regeneration of tubular epithelial cells after injury. ${ }^{10}$ In addition, Kim-1 expression has been reported to be low in the kidneys of young SHR (12 weeks of age), probably because of a malfunction in the ongoing renal maturation process. ${ }^{26}$ Consistently with these reports, this study showed that there was no detectable Kim-1 mRNA expression in salt-loaded SHR at 10 weeks of age, whereas Kim-1 was markedly upregulated at 17 weeks of age.

The origins of these urinary biomarkers might be renal tubules and/ or blood.

(1) Urinary vanin-1. Previous studies have shown that vanin-1 is mainly expressed in the kidneys, and vanin-1 is expressed in the epithelial cells of renal cortical tubules but not glomeruli. ${ }^{16}$ Previously, we also demonstrated that urinary vanin-1 was derived from renal proximal tubules in rats with nephrotoxicant- and drug-induced renal injury. ${ }^{17,18}$ In this study, the immunofluorescence analysis of vanin-1 also showed that vanin-1 is expressed in renal tubular cells in the saltloaded SHR. Therefore, urinary vanin-1 might be released from renal tubular epithelial cells in salt-loaded SHR. In hypertensive renal injury, hypertensive vascular damage occurs first in the juxtamedullary glomeruli, leading to impairment of renal mudullar circulation and medullary tubulointerstitial damage. ${ }^{27}$ The cortex is reported to be well protected from pressure-induced injury because renal cortical blood flow exhibits a high degree of autoregulation, and cortical renal damage is rarely found. ${ }^{27}$ Therefore, the tubulointerstitial damage and glomerular sclerosis in the cortex have been considered to be induced 


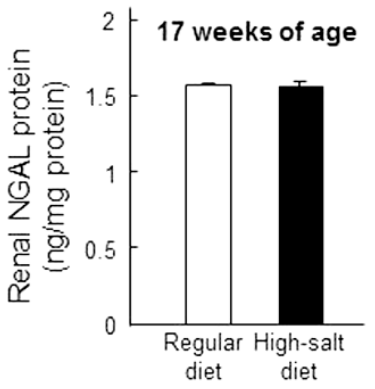

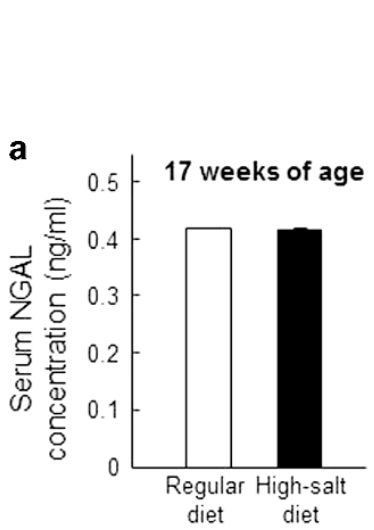

b Megalin
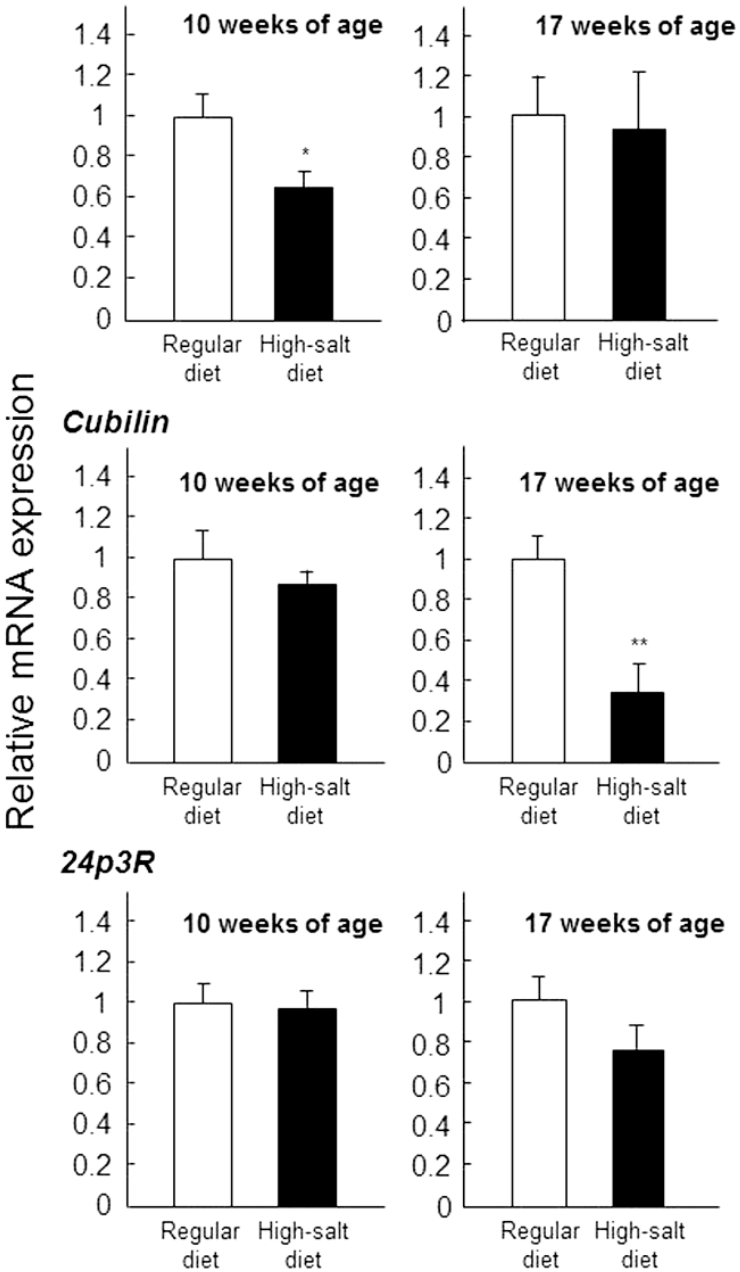

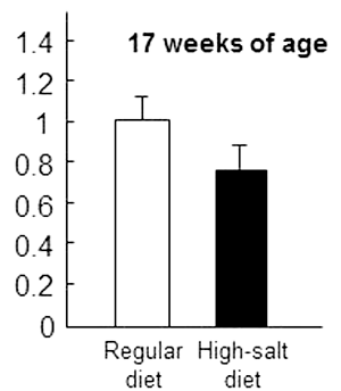

Figure 5 Origin of urinary NGAL. (a) Blood samples and kidney tissues were taken from animals at 17 weeks of age. Data are the means \pm s.e.m. ( $n=5-6$ ). (b) Kidney tissues were taken from animals at 9,10 and 17 weeks of age. The mRNA expression levels of megalin, cubilin and 24p3R were normalized to those of GAPDH. Data are the means \pm s.e.m. ( $n=4$ in each group). ${ }^{*} P<0.05 ;{ }^{*} P<0.01$ vs. SHR on a regular diet.

by a direct effect of intrarenal angiotensin II rather than by elevation of BP. Angiotensin II has been reported to initiate and enhance inflammation and oxidative stress. ${ }^{28,29}$ Because vanin-1 reacts with oxidative stress, and our results revealed that the cortex was stained positively for vanin-1 (Figure 4), urinary vanin-1 might originate from the injured tubules mainly in renal cortex in the salt-loaded SHR.

(2) Urinary NGAL. NGAL was detected in blood and kidney in this study. NGAL is a small protein $(21 \mathrm{kD})$ of the lipocalin superfamily and is filtered in urine. In renal proximal tubular epithelial cells, endocytosis receptors such as megalin, cubilin and $24 \mathrm{p} 3 \mathrm{R}$ are responsible for the reabsorption of glomerular-filtered proteins including NGAL. ${ }^{21,22,30}$ Megalin binds to cubilin with a high affinity and contributes to the internalization of cubilin-ligand complexes. ${ }^{31}$ Therefore, the impairment of these endocytosis receptors could lead to decreased renal uptake of NGAL. In this study, serum NGAL did not differ between the groups, but urinary NGAL was elevated in saltloaded SHR, which might have been caused by salt-induced decreases in the renal tubular megalin-cubilin complex.

(3) Urinary Kim-1. Blood and renal concentrations of Kim-1 and its urinary excretion were elevated in salt-loaded SHR at 17 weeks of age in this study. To evaluate the origin of urinary Kim-1, we conducted immunofluorescence analysis. Our results revealed that Kim-1 is localized mainly in the apical sides of renal tubules, which supports the idea that urinary Kim-1 is derived from the apical side of renal tubules. In this study, interstitial inflammation and fibrosis were marked in SHR on a high-salt diet at 17 weeks of age, whereas these changes were mild in SHR on a high-salt diet at 10 weeks of age. Of note, urinary excretions of vanin-1 and NGAL were elevated from 10 weeks of age, but urinary Kim-1 excretion was elevated from 15 weeks of age in SHR on a high-salt diet. These data indicate that interstitial inflammation and fibrosis are involved in the mechanism of the enhanced urinary Kim-1 in salt-loaded SHR.

Urinary albumin is reabsorbed via the megalin-cubilin complex. Therefore, the reabsorption of urinary albumin is potentially reduced in the salt-loaded SHR at 10 and 17 weeks of age. However, albumin has negative charge and a large molecular radius, and it is normally difficult to filter through the pores. ${ }^{32,33}$ Indeed, glomerulopathy was mild at 10 weeks of age (Supplementary Figure); thus, transglomerular passage of albumin may be low at 10 weeks of age. Therefore, the decrease in megalin gene expression was not reflected in urinary albumin excretion at 10 weeks of age. 
a

17 weeks of age

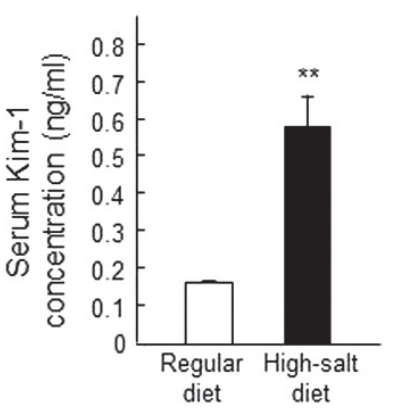

c

들

10 weeks of age

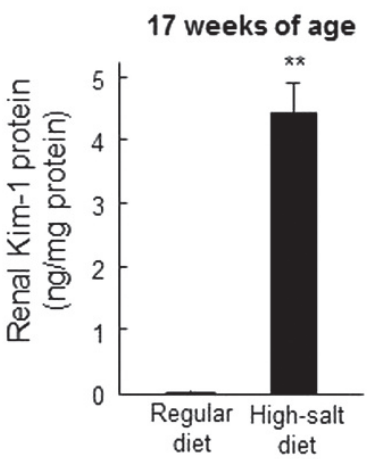

b

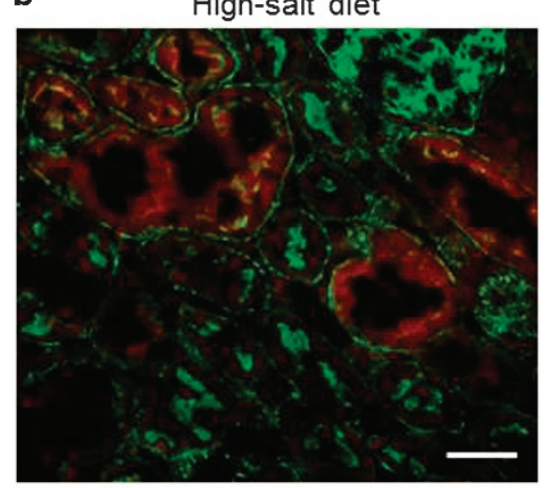

17 weeks of age

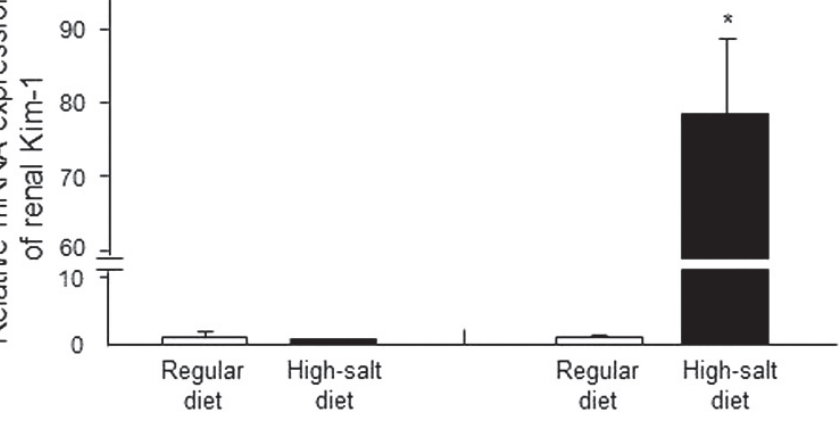

Figure 6 Origin of urinary Kim-1. (a) Blood samples and kidney tissues were taken from animals at 17 weeks of age. Data are the means \pm s.e.m. $(n=5-6)$. ${ }^{*} P<0.01$ vs. SHR on a regular diet. (b) A representative image of immunostaining of Kim-1 in the renal cortices of SHR on a high-salt diet. Cryosections $(4 \mu \mathrm{m})$ were stained with an antibody specific for Kim-1 (red) and phalloidin (green). Scale bars, $100 \mu \mathrm{m}$. (c) The mRNA expression levels of Kim-1 normalized to those of GAPDH. Data are the means \pm s.e.m. ( $n=4$ in each group).

Although BP was elevated ( $>200 \mathrm{~mm} \mathrm{Hg}$ ) in SHR on a regular diet at 17 weeks of age, renal tubular damage was relatively mild and was not reflected in the increases of urinary biomarkers in this study. These findings indicate that the role of salt might be greater than $t$ hat of BP elevation in the development of renal tubular damage in SHR on a high-salt diet. The following mechanisms might be involved in the high-salt-induced renal tubular damage observed in this study. Salt loading is known to suppress renin release from the renal juxtaglomerular apparatus and cause a reduction in systemically generated angiotensin II. However, Nath et al. ${ }^{34}$ have reported that, despite the suppression of circulating angiotensin II with salt loading, the locally generated intrarenal renin-angitensin-aldosteron system (RAAS) is stimulated by salt, and the angiotensin II content of proximal tubular fluid increases. Therefore, it is probable that the stimulated intrarenal RAAS is involved in salt-induced renal tubular damage.

Damage in the tubular epithelial cells causes tubulointerstitial injury and fibrosis, ${ }^{35}$ which is a common pathway for progression of many forms of renal disease. ${ }^{34}$ Clinically, it is important for detecting renal tubular damage at an early stage and initiating appropriate therapeutic intervention. The present study provides the hypothesis that urinary vanin-1 and NGAL might potentially be early biomarkers of renal tubular damage in hypertensive patients under a high-salt diet. To address this hypothesis, prospective clinical studies are required.

\section{CONFLICT OF INTEREST}

The authors declare no conflict of interest.

\section{ACKNOWLEDGEMENTS}

This work was supported by a Grant-in-Aid for Scientific Research (C) from the Ministry of Education, Culture, Sports, Science and Technology, Japan (26460631) and by a grant from the Ministry of Health, Labor and Welfare of Japan (H20-BIO-G003). K. Hosohata received research support from the Japan Research Foundation for Clinical Pharmacology.

1 Herlitz H, Dahlof B, Jonsson O, Friberg P. Relationship between salt and blood pressure in hypertensive patients on chronic ACE-inhibition. Blood Press 1998; 7: 47-52.

2 MacGregor GA, Markandu ND, Sagnella GA, Singer DR, Cappuccio FP. Double-blind study of three sodium intakes and long-term effects of sodium restriction in essential hypertension. Lancet 1989; 2: 1244-1247.

3 MacGregor GA. Salt: blood pressure, the kidney, and other harmful effects. Nephrol Dial Transplant 1998; 13: 2471-2479.

4 Ezzati M, Lopez AD, Rodgers A, Vander Hoorn S, Murray CJ. Selected major risk factors and global and regional burden of disease. Lancet 2002; 360: 1347-1360.

5 Jula AM, Karanko HM. Effects on left ventricular hypertrophy of long-term nonpharmacological treatment with sodium restriction in mild-to-moderate essential hypertension. Circulation 1994; 89: 1023-1031.

6 du Cailar G, Ribstein J, Mimran A. Dietary sodium and target organ damage in essential hypertension. Am J Hypertens 2002; 15: 222-229.

7 Teramoto T, Kawamori R, Miyazaki S, Teramukai S, Shirayama M, Hiramatsu K, Kobayashi F. Relationship between achieved blood pressure, dietary habits and cardiovascular disease in hypertensive patients treated with olmesartan: the OMEGA study. Hypertens Res 2012; 35: 1136-1144.

8 Ohta Y, Tsuchihashi T, Kiyohara K, Oniki H. High salt intake promotes a decline in renal function in hypertensive patients: a 10-year observational study. Hypertens Res 2013; 36: 172-176.

9 Mueller PW, Hall WD, Caudill SP, MacNeil ML, Arepally A. An in-depth examination of the excretion of albumin and other sensitive markers of renal damage in mild hypertension. Am J Hypertens 1995; 8: 1072-1082.

10 Ichimura T, Bonventre JV, Bailly V, Wei H, Hession CA, Cate RL, Sanicola M. Kidney injury molecule-1 (KIM-1), a putative epithelial cell adhesion molecule containing a 
novel immunoglobulin domain, is up-regulated in renal cells after injury. $J$ Biol Chem 1998; 273: 4135-4142.

11 Vaidya VS, Ramirez V, Ichimura T, Bobadilla NA, Bonventre JV. Urinary kidney injury molecule-1: a sensitive quantitative biomarker for early detection of kidney tubular injury. Am J Physiol Renal Physiol 2006; 290: F517-F529.

12 Han WK, Bailly V, Abichandani R, Thadhani R, Bonventre JV. Kidney Injury Molecule-1 (KIM-1): a novel biomarker for human renal proximal tubule injury. Kidney Int 2002; 62: 237-244.

13 Mishra J, Ma Q, Prada A, Mitsnefes M, Zahedi K, Yang J, Barasch J, Devarajan P. Identification of neutrophil gelatinase-associated lipocalin as a novel early urinary biomarker for ischemic renal injury. J Am Soc Nephrol 2003; 14: 2534-2543.

14 Mishra J, Mori K, Ma Q, Kelly C, Barasch J, Devarajan P. Neutrophil gelatinaseassociated lipocalin: a novel early urinary biomarker for cisplatin nephrotoxicity. Am J Nephrol 2004; 24: 307-315.

15 Aurrand-Lions M, Galland F, Bazin H, Zakharyev VM, Imhof BA, Naquet P. Vanin-1, a novel GPI-linked perivascular molecule involved in thymus homing. Immunity 1996; 5: 391-405.

16 Pitari G, Malergue F, Martin F, Philippe JM, Massucci MT, Chabret C, Maras B, Dupre S, Naquet P, Galland F. Pantetheinase activity of membrane-bound Vanin-1: lack of free cysteamine in tissues of Vanin-1 deficient mice. FEBS Lett 2000; 483: 149-154.

17 Hosohata K, Ando H, Fujiwara Y, Fujimura A. Vanin-1: a potential biomarker for nephrotoxicant-induced renal injury. Toxicology 2011; 290: 82-88.

18 Hosohata K, Ando H, Fujimura A. Urinary vanin-1 as a novel biomarker for early detection of drug-induced acute kidney injury. J Pharmacol Exp Ther 2012; 341: 656-662.

19 Matavelli LC, Zhou X, Varagic J, Susic D, Frohlich ED. Salt loading produces severe renal hemodynamic dysfunction independent of arterial pressure in spontaneously hypertensive rats. Am J Physiol Heart Circ Physiol 2007; 292: H814-H819.

20 Yu HC, Burrell LM, Black MJ, Wu LL, Dilley RJ, Cooper ME, Johnston CI. Salt induces myocardial and renal fibrosis in normotensive and hypertensive rats. Circulation 1998; 98: 2621-2628.

21 Christensen EI, Birn H. Megalin and cubilin: multifunctional endocytic receptors. Nat Rev Mol Cell Biol 2002; 3: 256-266.

22 Langelueddecke C, Roussa E, Fenton RA, Wolff NA, Lee WK, Thevenod F. Lipocalin-2 (24p3/neutrophil gelatinase-associated lipocalin (NGAL)) receptor is expressed in distal nephron and mediates protein endocytosis. J Biol Chem 2012; 287: 159-169.
23 Kitiyakara C, Chabrashvili T, Chen Y, Blau J, Karber A, Aslam S, Welch WJ, Wilcox CS. Salt intake, oxidative stress, and renal expression of NADPH oxidase and superoxide dismutase. J Am Soc Nephrol 2003; 14: 2775-2782.

24 Lai EY, Luo Z, Onozato ML, Rudolph EH, Solis G, Jose PA, Wellstein A, Aslam S, Quinn MT, Griendling K, Le T, Li P, Palm F, Welch WJ, Wilcox CS. Effects of the antioxidant drug tempol on renal oxygenation in mice with reduced renal mass. Am J Physiol Renal Physiol 2012; 303: F64-F74.

25 Berruyer C, Martin FM, Castellano R, Macone A, Malergue F, Garrido-Urbani S, Millet V, Imbert J, Dupre S, Pitari G, Naquet P, Galland F. Vanin-1-/- mice exhibit a glutathione-mediated tissue resistance to oxidative stress. Mol Cell Biol 2004; 24: 7214-7224.

26 Heijnen BF, Van Essen H, Schalkwijk CG, Janssen BJ, Struijker-Boudier HA. Renal inflammatory markers during the onset of hypertension in spontaneously hypertensive rats. Hypertens Res 2014; 37: 100-109.

27 Hultstrom M. Development of structural kidney damage in spontaneously hypertensive rats. J Hypertens 2012; 30: 1087-1091.

28 Kai H, Mori T, Tokuda K, Takayama N, Tahara N, Takemiya K, Kudo H, Sugi Y, Fukui D, Yasukawa H, Kuwahara F, Imaizumi T. Pressure overload-induced transient oxidative stress mediates perivascular inflammation and cardiac fibrosis through angiotensin II. Hypertens Res 2006; 29: 711-718.

29 Chen X, Mori T, Guo Q, Hu C, Ohsaki Y, Yoneki Y, Zhu W, Jiang Y, Endo S, Nakayama K, Ogawa S, Nakayama M, Miyata T, Ito S. Carbonyl stress induces hypertension and cardio-renal vascular injury in Dahl salt-sensitive rats. Hypertens Res 2013; 36: 361-367.

30 Hvidberg V, Jacobsen C, Strong RK, Cowland JB, Moestrup SK, Borregaard N. The endocytic receptor megalin binds the iron transporting neutrophil-gelatinase-associated lipocalin with high affinity and mediates its cellular uptake. FEBS Lett 2005; 579: 773-777.

31 Christensen EI, Verroust PJ, Nielsen R. Receptor-mediated endocytosis in renal proximal tubule. Pflugers Arch 2009; 458: 1039-1048.

32 Tencer J, Frick IM, Oquist BW, Alm P, Rippe B. Size-selectivity of the glomerular barrier to high molecular weight proteins: upper size limitations of shunt pathways. Kidney Int 1998; 53: 709-715.

33 D'Amico G, Bazzi C. Pathophysiology of proteinuria. Kidney Int 2003; 63: 809-825.

34 Nath KA. Tubulointerstitial changes as a major determinant in the progression of renal damage. Am J Kidney Dis 1992; 20: 1-17.

35 Healy E, Brady HR. Role of tubule epithelial cells in the pathogenesis of tubulointerstitial fibrosis induced by glomerular disease. Curr Opin Nephrol Hypertens 1998; 7: 525-530.

Supplementary Information accompanies the paper on Hypertension Research website (http://www.nature.com/hr) 